\title{
Stabilometry in Sports Medicine \& Doping Studies
}

\section{Hiroki Takada ${ }^{1,2 *}$}

${ }^{1}$ Department of Human \& Artificial Intelligent Systems, Graduate School of Engineering, University of Fukui, 3-9-1, Bunkyo, Fukui 910-8507, Japan ${ }^{2}$ Graduate School of Medicine, Aichi University of Medicine, Nagakute 480-1195, Japan

Keywords: Visually Induced Motion Sickness (VIMS); Sparse Density (SPD); Accommodation of lens; Convergence; Artificial gravity exposure; Electrooculography (EOG)

\section{Background and Objective}

Sports and/or doping are effective as a countermeasure for a broad range of problems in our daily lives. A countermeasure enables a recovery from some dysfunction or deconditioning such as temporary nearsightedness or space flight deconditioning. These two problems are outlined as follows.

\section{Myopia}

With the development of computers and the widespread use of the internet, an increasing number of people perform near-visual tasks such as operations on Video Display Terminals (VDTs). Working under these conditions for several hours induces contraction of the ciliary muscles, which are involved in focus adjustment around the eyeball. These muscles can abnormally contract as a result of the longterm performance of a near-visual task. This contraction causes various vision problems such as asthenopia and visual loss, and has been reported to induce cervicobrachial and psychoneurotic syndromes $[1,2]$. An accommodative function error of the lens causes these vision problems, which can be improved or prevented from progressing via ciliary muscle stretching exercises. These exercises can be performed by alternately repeating the negative and positive accommodations of the eye.

Some studies examined the effect of the operation of muscle activation, which is called accommodation training. For this, eyesight recovery and training apparatus (MD-SS type) was developed, which moves a Landolt ring drawn on a flat plate $2 \mathrm{~m}$ back and forth to encourage alternating negative and positive accommodation in the observers [3]. However, the moving distance of the target object is very short. Therefore, the back-and-forth motion of the objects might have little effect on the observers. In order to solve this problem while also reducing the size of the apparatus, alternative accommodation training was accomplished via images in three-dimensional (3D) movies. The $3 \mathrm{D}$ movies simulate the back-and-forth motion in a stereoscopic space by using a computer and a Liquid Crystal Display (LCD).

For persons afflicted with pseudo myopia, performing accommodation training alleviates strain and temporarily improves the myopic condition [4,5]. However, watching 3D movies may produce adverse effects such as asthenopia and motion sickness [6]. Measuring the severity of Visually Induced Motion Sickness (VIMS) can be accomplished by using psychological and physiological methods that have not been established. Our previous studies used the following hypothesis: VIMS changes the system to control the body sway [7-9].

\section{Space flight deconditioning}

It would be possible to prevent the harmful influence of microgravity exposure $\left(<10^{-6} \mathrm{G}\right)$ on the human body if a $1 \mathrm{G}$ environment were prepared in outer space [10]. Medical problems associated with longterm (greater than six months) microgravity exposure include "space motion sickness" in the early phases of space flight; cardiovascular deconditioning, which leads to decreased orthostatic tolerance; musculoskeletal deconditioning with atrophy, particularly in the antigravity muscles; and bone deconditioning with bone metabolism abnormalities. The general term used to describe these phenomena is "space deconditioning." Effective preventative measures for each type of deconditioning have been recommended, but comprehensive countermeasures have not been adopted.

Some devices have been developed as countermeasures using centrifuge-induced artificial gravity and ergometric exercise. Two types of devices are used to load artificial gravity: a long-arm device and a short-arm device. The former device can load 6-9 G onto subjects and is also used as a simulator for airplane pilots. However, it is difficult to load an artificial satellite or a space shuttle with a long-arm device. Therefore, the latter device, composed of a rotating rod with a diameter of $4 \mathrm{~m}$ that creates artificial gravity by centrifugal force and equipped with a bicycle ergometer, was created as a countermeasure against space deconditioning $[11,12]$. The anti-pooling function in the lower legs was significantly suppressed by a simulated microgravity exposure by using a $6^{\circ}$ head-down bed rest, and appeared to also be counteracted by centrifugal artificial gravity and ergonomic exercise [13].

Because of large individual differences in G-tolerance, anti-G tests were performed before and after microgravity exposure $[11,12]$. However, the effects of applied gravity as a countermeasure and gravitational loading in anti-G tests on vestibulospinal and vestibuloocular functions have not been evaluated. Therefore, in our study, we evaluated the effects of artificial gravity exposure on vestibulospinal and vestibulo-ocular functions using stabilometry [14].

\section{Solutions and recommendations}

The body's balance function utilizes sensory signals such as visual, auditory, and vestibular inputs, as well as proprioceptive inputs from the skin, muscles, and joints [15]. The evaluation of this function is indispensable for diagnosing equilibrium disturbances like cerebellar degenerations, basal ganglia disorders, or Parkinson's disease [16]. Stabilometry has been employed for both qualitative and quantitative evaluations of this equilibrium function. The projection of a subject's center of gravity onto a detection stand is measured as an average of the Center of Pressure (COP) of both feet. The COP is traced for each time step, and the time series of the projections is traced onto an $x-y$ plane. By connecting the temporally vicinal points, a stabilogram is created. Several parameters are commonly used in clinical studies to quantify the degree of instability while standing: area of sway (A), total locus length $(\mathrm{L})$, and locus length per unit area $(\mathrm{L} / \mathrm{A})$. The last parameter is strongly related to the fine variations involved in posture control [17].

*Corresponding author: Hiroki Takada, Department of Human \& Artificial Intelligent Systems, Graduate School of Engineering, University of Fukui, Japan, 910-8507, E-mail: takada@u-fukui.ac.jp

Received January 08, 2013; Accepted January 08, 2013; Published January 11 2013

Citation: Takada H (2013) Stabilometry in Sports Medicine \& Doping Studies. J Sports Med Doping Stud 3: e129. doi:10.4172/2161-0673.1000e129

Copyright: ( 2013 Takada H. This is an open-access article distributed under the terms of the Creative Commons Attribution License, which permits unrestricted use, distribution, and reproduction in any medium, provided the original author and source are credited. 
Thus, the $\mathrm{L} / \mathrm{A}$ index is regarded as a gauge for evaluating the function of proprioceptive control of standing. However, it is difficult to clinically diagnose balance disorders and identify a decline in equilibrium by utilizing the abovementioned indices and measuring patterns in a stabilogram. The large individual differences make it difficult to interpret the results of such a comparison.

For the data analysis, the anterior-posterior direction was considered to be independent of the lateral direction [18]. Stochastic Differential Equations (SDEs) were proposed as mathematical models to generate the stabilogram [19-21]. The variance in the stabilogram depends on the form of the temporally averaged potential function in the SDE, which generally has plural minimal points. In the vicinity of these points, local stable movement with a high-frequency component was generated as a numerical solution to the SDE. We can therefore expect a high density of observed COP in this area on the stabilogram [21]; Sparse Density (SPD) is regarded as an index for this measurement.

SPD is defined by an average of the ratio $G_{i}(1) / G_{j}(k)$ for $j=3,4$, ..., 20, where $G_{j}(k)$ is the number of divisions having more than $k$ measured points. A stabilogram is divided into quadrants whose latus is $j$ times longer than the resolution. If the center of gravity is stationary, the SPD value is 1 . If there are variations in the stabilograms, the SPD value is greater than 1 . Thus, the SPD depends on the characteristics of the stabilogram and the minimal structure of the temporally averaged potential function.

The forces acting on the center of gravity of the body are defined in terms of the differences in the displacement vectors. In particular, we focus on singular points at which statistically large forces are exerted. On the basis of these forces, chains are eliminated from the stabilogram in the form of a consecutive time series. If the times measured at these points were in the temporal vicinity, these points were connected by segments (sequences). Figures formed by these sequences are called "chains" because of the shape of the connections. The figures demonstrate that the chains at which large forces are exerted have cusp patterns.

\section{Future research directions}

These geometrical indices in stabilograms have already been calculated out of physiological curiosity to evaluate equilibrium deterioration with advancing age [22] and cerebral suppression from alcoholic intake $[23,24]$. However, the analytical results of the calculation of the indices can also be applied to develop devices that reduce the severity of motion sickness induced by blurred images on LCDs [7], $3 \mathrm{D}$ video clips [8,9], artificial gravitational loads [17], and Galvanic Vestibular Stimulations (GVS), which mask regular signals until they reach vestibular nuclei [25]. The use of GVS during simulated driving may create a highly realistic experience, supporting the contention that the application of GVS during simulations in a virtual environment reduces simulator adaptation syndrome [26]. Observation of multiple senses may assist our investigations and developments.

Why are stereoscopic images unnatural for human vision? According to a commonly accepted explanation, a certain sensory conflict causes problems while viewing stereoscopic images. A general explanation is as follows: "During stereoscopic vision, accommodation and convergence are mismatched and this is the main reason for the visual fatigue caused by $3 \mathrm{D}$. During stereoscopic vision, while accommodation is fixed on the display that shows the 3D image, convergence of left and right eyes crosses at the location of the stereo image."

Patterson also provided a recent estimate of the depth of field of the human eye and shows that the accommodation-convergence conflict would likely not occur under most stereo display viewing conditions, i.e., it might only occur when using near-eye displays [27]. The critical issue is to present the virtual images at depth planes that fall within the depth of field of the viewer, which would prevent such conflict; this would likely happen under most conditions. When the virtual image is presented within the viewer's depth of field, the accommodation can follow the convergence response without any conflict because there would be no blur signal to drive the accommodation back to the display surface. Under such conditions, it would be predicted that the viewer would accommodate at the distance of the convergence response, for which support is provided by our previous studies [28,29].

A sensory conflict between the visual and vestibular systems caused increased sway with eyes open during stabilometry after graded gravitational loading [14]. To confirm these findings, we measured eye movements using EOG in order to evaluate the vestibular nystagmus after initiation and discontinuation of centrifugal rotation. The analysis of the EOG results demonstrated the conflict between the visual and vestibular information. Next, optokinetic after-nystagmus and postrotatory nystagmus induced by the vestibulo-ocular reflex must be investigated in detail using an infrared charge-coupled device camera or Frenzel glasses. Moreover, we will examine whether the artificial gravity with ergometric exercise can be regarded as a countermeasure of space flight deconditioning in accordance with the bedrest studies.

\section{References}

1. Matsuura Y, Amemori M, Shiomi T, Miyao M, Yoshikawa K, et al. (2002) Association between duration of daily VDT use and subjective symptoms. Am $\mathrm{J}$ Ind Med 42: 421-426.

2. Gomzi M (1994) Work environment and health in VDT use: An ergonomic approach. Arh Hig Rada Toksikol 45: 327-334.

3. Kobayashi S (1994) an MD-SS eyesight recovery and training apparatus Japan Patent 6: 339-501.

4. Sugiura A, Miyao M, Yamamoto T, Takada H (2011) Effect of strategic accommodation training by wide stereoscopic movie presentation on myopic young people of visual acuity and asthenopia. Displays 32: 219-224.

5. Takada M, Miyao M, Satoh M, Yoshikawa K, Matsuura Y, et al. (2012) Effect of accommodation training on visual function of visual inspection workers and middle-aged people. J Sports Med Doping Stud 2: 3.

6. Takada H, Fujikake K, Miyao M, Matsuura Y (2007) Indices to detect visually induced motion sickness using stabilometry. Proceedings of VIMS2007: 178183.

7. Fujikake K, Takada H, Omori M, Miyao M (2008) Evaluation of high-quality LCDs displaying moving pictures by use of the form obtained from statokinesigrams and the dynamics. Forma 22: 217-229.

8. Takada H, Miyao M, Takada M, Matsuura Y, Yamamoto T (2012) Effect of stereoscopic movie on body sway and head acceleration. Computer Technology and Application 3: 159-168

9. Takada H, Miyao M (2012) Visual Fatigue and Motion Sickness Induced by 3D Video Clip. Forma 27(S) (In Press)

10. Sekiguchi C (1998) Space medicine: physiology. Shakaihoken Press; Tokyo 22-23

11. Iwase S, Takada H, Watanabe Y, Ishida K, Akima H, et al. (2004) Effect of centrifuge-induced artificial gravity and ergometric exercise on cardiovascular deconditioning myatrophy, and osteoporosis induced by a -6 degrees headdown bedrest. J Gravit Physiol 11: 243-244.

12. Iwase S, Fu Q, Narita K, Morimoto E, Takada H, et al. (2002) Effects of graded load of artificial gravity on cardiovascular functions in humans. Environ Med 46: 29-32.

13. Watanabe Y, Takada H, Iwase S, Fu Q (2003) Effect of microgravity exposure on post-ischemic hyperemia at the forearm and calf, and influence of artificia gravity with ergometric exercise as a countermeasure. Autonomic Nervous System 40: 488-494

14. Yamamoto T, Takada H, Iwase S, Sugenoya J (2011) Effects of graded load 
of artificial gravity on vestibulospinal and vestibule-ocular functions. Autonomic Nervous System 47: 485-491.

15. Kaga K, Memaino K (1992) Structure of vertigo, Tokyo: Kanehara.

16. Okawa T, Tokita T, Shibata Y, Ogawa T, Miyata H (1995) Stabilometry: Significance of locus length per unit area (L/A). Equilibrium Res 54: 296-306.

17. Okawa T, Tokita T, Shibata Y, Ogawa T, Miyata H (1995) Stabilometry: Significance of locus length per unit area (L/A) in patients with equilibrium disturbances. Equilibrium Res 55: 283-293.

18. Goldie PA, Bach TM, Evans OM (1986) Force platform measures for evaluating postural control: reliability and validity. Arch Phys Med Rehabil 70: 510-517.

19. Collins JJ, De Luca CJ (1993) Open-loop and closed-loop control of posture: A random-walk analysis of center-of-pressure trajectories. Exp Brain Res 95: 308-318.

20. Newell KM, Slobounov SM, Slobounova ES, Molenaar PC (1997) Stochastic processes in postural center-of-pressure profiles. Exp Brain Res 113: 158-164.

21. Takada H, Kitaoka Y, Shimizu Y (2001) Mathematical index and model in stabilometry. Forma 16: 17-46.

22. Takada H, KitaokaY, Iwase S, Shimizu Y, Watanabe T, et al. (2003) Characteristic changes of sway of center of gravity with advancing afe. Env Med 47: 85-89.

23. Takada H, Kitaoka Y, Ichikawa M, Miyao M (2003) Physical meaning on geometrical index for stabilometry. Equilibrium Research 62: 168-180.
24. Matsuura Y, Amemori M, Shiomi T, Miyao M, Yoshikawa K, et al. (2012) Evaluation of body sway in a seated posture after alcohol ingestion with an aim to evaluate motion sickness caused by three-dimensional images. J Sports Med Doping Stud 2: 2

25. Takada H, Takada M, Tanaka K, Shiozawa T, Furuta M, et al. (2009) A simulated study of the deterioration in the equilibrium function With advancing age Bulletin of Gifu University of Medical Science 3: 109-117.

26. Jones RJR, Jones JGR, Trick LM, Vallis LA (2007) Can galvanic vestibular stimulation reduce simulator adaptation syndrome? Proceedings of the fourth international driving symposium on human factors in drive assessment, training and vehicle Design 534-540.

27. Patterson R (2009) Human factors of stereo displays: An update. J Soc Inf Disp 17: 987-996.

28. Miyao M, Shiomi T, Hori H, Hasegawa A, Matsuura Y, et al. (2011) Comparison of $2 \mathrm{D}$ and $3 \mathrm{D}$ vision gaze with simultaneous measurements of accommodation and convergence among young and middle-aged subjects. IMID Digest 149 150 .

29. Kojima T, Matsuura Y, Miyao M, Shiomi T, Takada H (2012) 'Comparison by simultaneous measurement of lens accommodation and convergence in 3D vision and their distributions'. International Journal of Bioscience, Biochemistry and Bioinformatics. 